\title{
Preparação e Caracterização de Organobentonita Modificada com Ionenos Alifáticos
}

\author{
Arão P. Costa Filho \\ Instituto de Macromoléculas Professora Eloisa Mano, UFRJ \\ Departamento de Tecnologia Química, Universidade Federal do Maranhão
}

\author{
Ailton S. Gomes, Elizabete F. Lucas \\ Instituto de Macromoléculas Professora Eloisa Mano, UFRJ
}

\begin{abstract}
Resumo: A preparação de complexos bentonita-ionenos a partir da adsorção de tetrametil-[x,y]-ionenos, onde $\mathrm{x}=2$ e $\mathrm{y}=$ 4,6,10 ou 12, e o 2-hidróxi-3-cloro-ioneno, EPI-DMA, foi realizada com a finalidade de observar o comportamento do tamanho do espaçador do ioneno na expansão basal $\left(\mathrm{d}_{001}\right)$ das camadas de uma bentonita comercial do Brasil. Os complexos foram caracterizados por espectrometria de absorção na região do infravermelho, termogravimetria, fluorescência e difração de raios X. A partir das análises por fluorescência e difração de raios X dos complexos, foi observado que os poliquaternários de amônio são adsorvidos nas superfícies internas e externas da argila delaminada, acima da capacidade de troca catiônica, CTC $=91 \mathrm{meq} / 100 \mathrm{~g}$ de argila (calculada a partir de dados de fluorescência de raios X). A expansão basal das camadas da argila com os diferentes policátions revelou que o tamanho do espaçador é o fator mais importante, independentemente das massas molares dos ionenos, tal como foi observado principalmente entre os complexos Bt-2,10e Bt-2,12-ioneno, com massas molares de 12000 e $5000 \mathrm{~g} / \mathrm{mol}$, respectivamente.
\end{abstract}

Palavras-chave: Ionenos, organobentonitas, espaçadores parafinicos, poluentes orgânicos.

\section{Preparation and Characterization of Aliphatic Ionene-Modified Organobentonite}

Abstract: The preparation of bentonite-ionene complexes based on the adsorption of 2y-ionenes, where $y=4,6,10$ or 12 and an ionene of the epichloridrin-dimethylamine EPI-DMA kind, was carried out in order to observe the behavior of the ionene spacer size in the expansion of the basal space (d-spacing, $\mathrm{d}_{001}$ ) of a Brazilian commercial bentonite. The complexes were characterized by adsorption spectromety in the infrared region, thermogravimetry, fluorescence and X-Ray diffraction. Ionenes were synthesized and characterized by infrared and nuclear magnetic resonance, the latter technique being useful for determining the average number molecular weight values. Based on the clay-ionenes complexes it could be observed that the ammonium polyquaternaries are adsorbed on the inner and outer clay surfaces, above the ion exchange capacity, $\mathrm{CEC}=91 \mathrm{meq} / 100 \mathrm{~g}$ clay (calculated based on X-Ray fluorescence data). The clay basal expansion with the several polycations revealed that the spacer size is the most important factor, independent of ionene molecular weight values. Among the bentonite-ionene complexes, mainly between Bt-2,10 and Bt-2,12-ionene complexes which differ in molar mass by more than $30,000 \mathrm{~g} / \mathrm{mol}$, it was observed that the spacer size has no dependence on the ionene molecular weights.

Keywords: Ionene polymers, organobentonite, hydrocarbon spacers, pollutants.

\section{Introdução}

A bentonita é um argilomineral composto principalmente de montmorilonita, que é um aluminossilicato do tipo $2: 1$, ou seja, sua estrutura cristalina apresenta uma camada octaédrica de alumina entre duas camadas octaédricas de sílica (Figura 1), que por um processo de substituições isomórficas, denominado troca catiônica, requer cátions para compensar as cargas negativas das extremidades de suas camadas. A bentonita tem a capacidade de trocar es- ses cátions com outros presentes em soluções aquosas de sais orgânicos ou inorgânicos. Quando na troca catiônica são usadas soluções de cátions orgânicos, como os quaternários de amônio, a bentonita modificada é chamada de organobentonita ou bentonita organofílica.

Argilas modificadas com sais monoquaternários de amônio de comprimentos de cadeia variados têm sido investigadas quanto às suas capacidades na remoção de diversos tipos de poluentes orgânicos, tais como, organoclorados $^{[1,2]}$, óleo e graxa ${ }^{[3]}$, dodecilbenzeno sulfonato de

Autor para correspondência: Ailton S. Gomes, Instituto de Macromoléculas Professora Eloísa Mano, UFRJ, Caixa Postal 68525, CEP: $21945-570$, Rio de Janeiro, RJ. E-mail: asgomes@ima.ufrj.br 


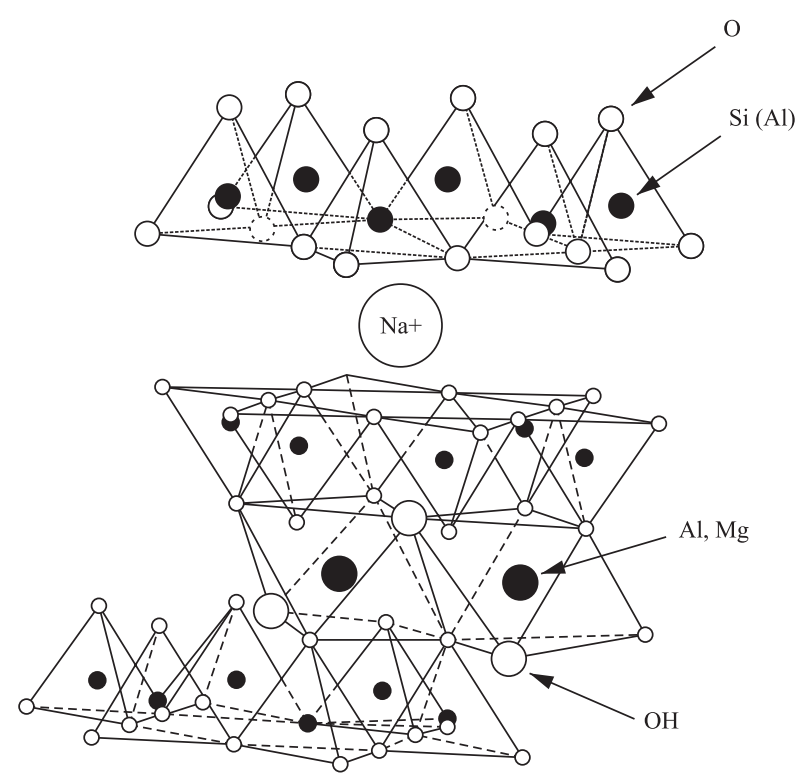

Figura 1. Estrutura química da montmorilonita

sódio $^{[4]}$, gasolina ${ }^{[5]}$, benzeno ${ }^{[6]}, 2$-nafto ${ }^{[7]}$ e naftaleno ${ }^{[8,9]}$.

Os cátions orgânicos pequenos (tetrametilamônio) geram superfícies apolares relativamente rígidas, responsáveis pela remoção, por adsorção, de solutos não-iônicos. Cátions orgânicos maiores (hexadecil-trimetilamônio) adsorvidos na argila, geram um meio orgânico de partição através da aglomeração de suas cadeias alquílicas flexíveis ${ }^{[10]}$. Quando um policátion é adicionado à superfície de uma argila, o contato entre ele e a superfície acontece de forma que a configuração da cadeia polimérica adquire vários segmentos "trens" em contato íntimo com a superfície alternada por "laços" e produzindo "caudas" nas extremidades da cadeia (Figura 2), os quais não estão adsorvidos. Este processo acarreta a remoção de grandes quantidades de água e cátions inorgânicos trocáveis da argila. A interação entre o policátion e a superfície da argila ocorre via interações coulômbicas entre os sítios de carga positiva no polímero e a superfície da argila carregada negativamente ${ }^{[11]}$. A adsorção de um policátion é um processo irreversível. Foi demonstrado que policátions adsorvidos nas superfícies de argilas desenvolvem uma rede de carga positiva e também as deixam aptas para remover poluentes orgânicos ${ }^{[12-14]}$.

Estudos realizados com o 2-hidróxi-3-cloro-ioneno, EPIDMA, mostraram que ele foi segregado em diferentes intercamadas da argila em quantidades de $30 \%$ abaixo da sua capacidade de troca catiônica (CTC). Também foi verificado

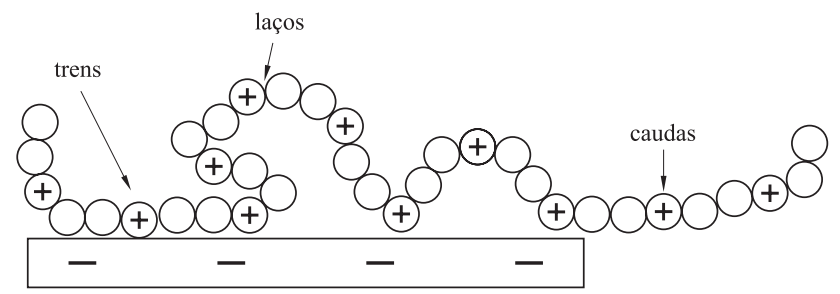

Figura 2. Elementos conformacionais da cadeia de um policátion adsorvido na superfície de uma argila.<smiles>CCCC[N+](C)(C)C[N+](C)(C)C[N+](C)(C)CC(O)CC</smiles>

(a)

(b)

Figura 3. Fórmula geral dos ionenos sintetizados.

que ele apresentou melhor poder absorvente para o pnitrofenol que o cloreto de poli-dialildimetilamônio (poliDADMAC) $)^{[15]}$.

Os tetrametil-[x,y]-ionenos são poli-quaternários de amônio lineares com o átomo de nitrogênio quaternário inserido na cadeia polimérica. As fórmulas estruturais para os tetrametil-ionenos e para o ioneno EPI-DMA estão representadas na Figura 3. O termo ioneno foi introduzido por Rembaum pelo fato desses polímeros serem sintetizados a partir da ionização de aminas ${ }^{[16]}$. A semelhança estrutural entre os tetrametil-[x,y]-ionenos e EPI-DMA nos levou a investigar organobentonitas modificadas com esses polímeros por se tratarem de policátions onde a densidade de carga e o tamanho da cadeia hidrofóbica podem ser facilmente variados.

O objetivo deste trabalho foi usar uma quantidade fixa de policátion $(0,66 \mathrm{~g}$ de polímero/g de argila) e saturar as superfícies de uma bentonita comercial com capacidade de troca catiônica (CTC) de $91 \mathrm{meq} / 100 \mathrm{~g}$. Em seguida, verificar a influência dos espaçadores desses policátions nas distâncias interplanares, quando eles são adsorvidos nas superfícies dessa bentonita, para posteriormente avaliar o potencial desses complexos formados como materiais capazes de remover poluentes orgânicos de águas residuais.

\section{Experimental}

$\mathrm{N}, \mathrm{N}, \mathrm{N}^{\prime}, \mathrm{N}^{\prime}$-Tetrametiletilenodiamina (TMEDA), 1,4dibromobutano (1,4-DBB), 1,10-dibromodecano (1,10-DBD) e 1,12-dibromododecano (1,12-DBDD) foram obtidos da Sigma-Aldrich do Brasil, São Paulo,Brasil; 1,6-dibromohexano (1,6-DBH), solução aquosa a $60 \%$ de dimetilamina (DMA) (doada pela BASF); metanol $(\mathrm{MeOH})$ grau espectroscópico e N,N-dimetilformamida (DMF), adquiridos da Merck, São Paulo, Brasil, e a bentonita sódica (Bt-Na) comercial foi obtida da Bentonita do Brasil S.A. Todos esses produtos foram utilizados como recebidos.

\section{Síntese e caracterização dos ionenos e do composto de referência}

Os ionenos foram sintetizados de acordo com a literatu$\mathrm{ra}^{[17]}$. O ioneno EPI-DMA foi sintetizado a partir da adição de epicloridrina (EPI) a uma solução aquosa a $60 \%$ de dimetilamina (DMA $)^{[18]}$.

\section{Espectroscopia de ressonância magnética nuclear}

Os ionenos foram caracterizados estruturalmente por ressonância magnética nuclear de hidrogênio $\left(\mathrm{NMR}-\mathrm{H}^{1}\right)$, em 
$\mathrm{D}_{2} \mathrm{O}$, utilizando um espectrômetro de ressonância magnética nuclear operando na freqüência de $300 \mathrm{MHz}$, modelo VARIAN Gemini 300. Essa técnica também permitiu a obtenção dos pesos moleculares de todos os ionenos ${ }^{[19]}$.

\section{Preparação e caracterização dos complexos bentonita- ionenos}

$\mathrm{Na}$ preparação dos complexos bentonita-ionenos (Btionenos), foram utilizados $9 \mathrm{~g}$ de bentonita sódica (Bt-Na). A argila foi delaminada em $200 \mathrm{~mL}$ de água deionizada, sob agitação à temperatura ambiente. Após 10 minutos de agitação, foi adicionada uma solução aquosa do ioneno de concentração a $6 \% \mathrm{p} / \mathrm{v}$ e a agitação permaneceu por mais dez minutos. A mistura obtida foi transferida para uma proveta com tampa e deixada sob repouso por 24 horas. A mistura foi, então, filtrada a vácuo e lavada com água deionizada até não indicar mais íons brometo, pela adição de solução de $\mathrm{AgNO}_{3} \mathrm{e}$, em seguida, foi lavada com etanol comercial e colocada para secar entre $60-65{ }^{\circ} \mathrm{C}$ por 48 horas. O material seco foi triturado em um gral, passado em peneiras ABNT $\mathrm{N}^{\circ} 200(\mathrm{D}=74 \mu \mathrm{m})$ e analisado por fluorescência de raios $\mathrm{X}$, espectroscopia de absorção na região do infravermelho (FT-IR), termogravimetria (TG) e difração de raios-X. Essa operação foi repetida para cada um dos ionenos aqui estudados.

Com o objetivo de verificar as mudanças ocorridas na argila após o tratamento com os ionenos, a bentonita sódica Bt$\mathrm{Na}$ e os complexos Bt-ionenos foram caracterizados para verificação da composição, estabilidade térmica e espaçamento basal.

\section{Fluorescência de raios $\mathbf{X}$}

Os sistemas foram analisados por fluorescência de raios X com o método de fusão de $\mathrm{LiB}_{4} \mathrm{O}_{7}$, em um equipamento Rigaku, modelo RIX3100 com tubo de ródio. Essa técnica também permitiu calcular a capacidade de troca catiônica da bentonita, cujo valor é de $91 \mathrm{meq} / 100 \mathrm{~g}$ de argila.

\section{Termogravimetria}

A termogravimetria dos complexos Bt-ionenos foi realizada na faixa de 30 a $800{ }^{\circ} \mathrm{C}$, em uma razão de aquecimento de $10{ }^{\circ} \mathrm{C} /$ minuto sob atmosfera de nitrogênio, utilizando um analisador termogravimétrico modelo TAC7/DX, Perkin Elmer.

\section{Espectroscopia de absorção na região do infravermelho}

Foi utilizado um espectrômetro de absorção na região do infravermelho com transformada de Fourier - modelo 1720X PE, Perkin Elmer. As amostras foram analisadas na forma de pastilhas de $\mathrm{KBr}$ dentro da faixa de freqüência de 4000 a $400 \mathrm{~cm}^{-1}$ com resolução de $4 \mathrm{~cm}^{-1}$.

\section{Espectroscopia fotoeletrônica de raios $\mathbf{X}$}

Os sistemas foram analisados por difração de raios $\mathrm{X}$ utilizando um equipamento Rigaku da marca Philips, modelo Miniflex Goniometer, utilizando radiação $\mathrm{K} \alpha$ do cobre $(\lambda=1,5418 \mathrm{~nm})$.

\section{Resultados e Discussão}

\section{lonenos}

Cada ioneno sintetizado foi caracterizado para verificação da sua estrutura e massa molar.

Tabela1. Dados de caracterização por ressonância magnética nuclear de hidrogênio para os ionenos

\begin{tabular}{|c|c|c|c|c|c|}
\hline$\sigma($ ppm)/A(u.a) & 2,4-ioneno & 2,6-ioneno & 2,10-ioneno & 2,12-ioneno & HICP \\
\hline $\mathrm{C}-\left(\mathrm{CH}_{2}\right)_{2}-\mathrm{C}$ & $1,97 / 15,78$ & - & - & - & - \\
\hline $\mathrm{C}_{2}-\left(\mathrm{CH}_{2}\right)_{2}-\mathrm{C}_{2}$ & - & $1,46 / 11,48$ & - & - & - \\
\hline $\mathrm{C}-\left(\mathrm{CH}_{2}\right)-\mathrm{C}_{2}-\left(\mathrm{CH}_{2}\right)-\mathrm{C}$ & - & $1,85 / 11,14$ & - & - & - \\
\hline $\mathrm{C}-\left(\mathrm{CH}_{2}\right)-\mathrm{C}_{6}-\left(\mathrm{CH}_{2}\right)-\mathrm{C}$ & - & - & $1,78 / 11,19$ & - & - \\
\hline $\mathrm{C}_{2}-\left(\mathrm{CH}_{2}\right)_{6}-\mathrm{C}_{2}$ & - & - & $1,33 / 32,87$ & - & - \\
\hline $\mathrm{C}-\left(\mathrm{CH}_{2}\right) \mathrm{C}_{8}\left(\mathrm{CH}_{2}\right)-\mathrm{C}$ & - & - & - & $2,28 / 8,06$ & - \\
\hline $\mathrm{C}_{2}-\left(\mathrm{CH}_{2}\right)_{8}-\mathrm{C}_{2}$ & - & - & - & $1,77-1,84 / 31,15$ & - \\
\hline $\mathrm{CH}(\mathrm{OH})$ & - & - & - & - & $3,77 / 18,04$ \\
\hline $\mathrm{N}^{+}-\left(\mathrm{CH}_{3}\right)_{2}$ & $3,27 / 44,12$ & $3,22 / 31,98$ & $3,18 / 31,48$ & $3,68 / 23,2$ & $3,34 / 34,16$ \\
\hline $\mathrm{N}^{+}-\mathrm{CH}_{2}$ & $3,60 / 17,93$ & $3,46 / 11,58$ & $3,41 / 12,81$ & $3,90 / 9,41$ & $3,60 / 18,78$ \\
\hline $\mathrm{N}^{+} \mathrm{CH}_{2} \mathrm{CH}_{2} \mathrm{~N}^{+}$ & $4,03 / 15,79$ & $3,95 / 11,24$ & $3,91 / 10,62$ & $4,36 / 8,26$ & - \\
\hline $\mathrm{N}\left(\mathrm{CH}_{3}\right)_{2}$ & $2,67 / 1,02$ & $2,27 / 0,76$ & $2,69 / 1,04$ & $3,22 / 2,03$ & $1,5 / 0,29$ \\
\hline
\end{tabular}




\section{Caracterização estrutural}

Espectroscopia de ressonância magnética nuclear

As estruturas dos ionenos foram confirmadas por meio de ressonância magnética nuclear de hidrogênio. Os valores de deslocamento químico e áreas integradas $\sigma(\mathrm{ppm}) / \mathrm{A}($ u.a), respectivamente, são dados na Tabela 1. Os sinais observados para os ionenos e suas respectivas áreas foram utilizados para a determinação do peso molecular.

\section{Determinação da massa molar $\left(\bar{M}_{\mathrm{n}}\right)$ por $\mathbf{R M N}-\mathrm{H}^{\mathbf{1}}$}

O conhecimento da massa molar dos polímeros preparados é de grande importância para verificar sua influência nas propriedades dos complexos Bt-ionenos. O grau de polimerização $\bar{X}_{n}$, dos ionenos foi determinado através da razão entre as áreas dos picos referentes aos hidrogênios metílicos ligados aos grupos quaternários de amônio na cadeia e o grupo dimetilamina terminal de cadeia ${ }^{[19]}$, de acordo com as Equações 1 e 2:

$$
\bar{X}=\mathrm{A}_{\mathrm{N}(\mathrm{CH} 3) 2}^{+} / \mathrm{A}_{\mathrm{N}(\mathrm{CH} 3) 2}
$$

onde A é a área da integração dos picos. A massa molar foi estimada por:

$$
\bar{M}_{n}=\bar{X}_{n} \mathrm{xPM}_{(\mathrm{ur})}
$$

onde $\mathrm{PM}_{(\mathrm{u} . \mathrm{r})}$ é a massa molar da unidade polimérica repetitiva. Os valores de massa molar obtidos para 2,4-, 2,6-, 2,10 e 2,12-ioneno foram $13000,15000,12000$ e $5000 \mathrm{~g} / \mathrm{mol}$, respectivamente.

A determinação da massa molar através de $\mathrm{RMN}-\mathrm{H}^{1}$ é particularmente vantajosa para esses polímeros de baixa massa molar, desde que se disponha de um espectro isento de ruídos e picos de impurezas, sobretudo quando esses picos estão sobrepostos àqueles correspondentes aos hidrogênios do policátion. Ionenos de diferentes massas molares podem ser obtidos sob condições reacionais variadas ${ }^{[16]}$.

\section{Complexos Bt-ionenos}

É mencionado na literatura que sistemas semelhantes aos deste trabalho não apresentam influência da massa molar sobre o espaçamento basal de seus complexos com bentonita sódica $^{[12]}$. Assim, foi utilizada uma amostra de cada tipo de ioneno, independente do peso molecular, para preparar os complexos com bentonita sódica.

Durante a preparação dos complexos Bt-ionenos, foi observada uma variação na maleabilidade dos complexos formados de acordo com o tamanho do espaçador. Na etapa de desagregação, os menores espaçadores geraram complexos que exigiram um maior esforço de desagregação (Bt-EPIDMA e Bt-2,4 ioneno), ao contrário dos Bt-2,10 e Bt-2,12 ionenos, os quais foram mais fáceis de serem desagregados.

\section{Fluorescência de raios $\mathrm{X}$}

Através da análise de fluorescência de raios $\mathrm{X}$ foi possível obter dados semiquantitativos da composição elementar da bentonita sódica e dos complexos Bt-ionenos (Tabela 2).
Foi detectada a presença de aproximadamente $6 \%$ de carbono, cuja origem é desconhecida. Em todos os casos, foi observado que a adição de soluções de polímeros às suspensões de argila acarretou em aumento expressivo do teor de carbono nos complexos obtidos. $\mathrm{O}$ alto grau de incorporação dos ionenos pôde ser confirmado pela redução dos teores de sódio e cálcio na bentonita sódica, evidenciando sua remoção pelos policátions. Por se tratar de uma argila sódica, os percentuais de íons $\mathrm{Na}^{+}$trocados ocorreram com média de 95\%, mostrando que esses polímeros possuem grande afinidade pela bentonita sódica, independente do tamanho do espaçador e da massa molar dos policátions.

O percentual de cloreto na amostra de Bt-EPI-DMA confirma um excesso de carga positiva no sistema, indicando que a troca catiônica foi satisfatória, o que foi observado quando esse mesmo ioneno foi adsorvido em uma bentonita do Texas de baixo teor de ferro ${ }^{[15]}$. De forma semelhante, foram detectados contra-íons $\mathrm{Br}^{-}$associados aos grupos quaternários de amônio não adsorvidos na superfície da argila, provavelmente localizados em segmentos de laços ou caudas na cadeia do policátion, confirmando que a troca catiônica também foi satisfatória nesses sistemas.

Já é conhecido da literatura, que polímeros com características estruturais semelhantes aos ionenos, adsorvem de forma intensa em argilas sódicas ${ }^{[14]}$, inclusive o EPI-DMA, o qual também apresentou afinidade acentuada por bentonitas sódicas em frações muito finas ${ }^{[15]}$.

\section{Espectroscopia de absorção na região do infravermelho}

Os espectros de infravermelho mostraram bandas comuns em aproximadamente $3623 \mathrm{~cm}^{-1,} 3410 \mathrm{~cm}^{-1}, 1614 \mathrm{~cm}^{-1}$ referentes às vibrações complexas do grupo $\mathrm{OH}$ [20]. Além das bandas observadas para a $\mathrm{Bt}-\mathrm{Na}$, os espectros dos complexos mostraram bandas em $1471 \mathrm{~cm}^{-1}$, devido à vibração de deformação angular simétrica no plano de $\mathrm{CH}_{2}$, em $2852 \mathrm{~cm}^{-1}$, $2940 \mathrm{~cm}^{-1}$ e $3010 \mathrm{~cm}^{-1}$ devido à deformação axial assimétrica de $\mathrm{CH}_{3}$ e $\mathrm{CH}_{2}$. Absorções em $2940 \mathrm{~cm}^{-1}$ e $3010 \mathrm{~cm}^{-1}$ não foram evidenciadas no espectro do Bt-EPI-DMA, pois a absorção do grupo $\mathrm{OH}$ nesta mesma região provoca um alargamento da banda. Essas bandas observadas para os complexos confirmam a intercalação dos ionenos nos espaços interlamelares da bentonita. Um comportamento semelhante

Tabela 2. Análises de fluorescência de raios X (em \% de massa)

\begin{tabular}{lccccccc}
\hline Composto & $\mathbf{C}$ & $\mathbf{N a}$ & $\mathbf{M g}$ & $\mathbf{A l}$ & $\mathbf{S i}$ & $\mathbf{B r}$ & $\mathbf{C a}$ \\
\hline Bt-Na & 5,81 & 2,81 & 4,52 & 14,6 & 57,9 & - & 2,22 \\
Bt-HICP & 11,3 & 0,08 & 3,28 & 9,59 & 60,3 & $2,57(\mathrm{Cl})$ & 0,72 \\
Bt-2,4 & 10,4 & 0,11 & 2,69 & 7,93 & 58,1 & 7,79 & 0,85 \\
Bt-2,6 & 14,5 & 0,09 & 2,82 & 7,76 & 57,4 & 3,76 & 1,10 \\
Bt-2, 10 & 16,6 & 0,20 & 2,72 & 7,20 & 55,2 & 5,32 & 1,30 \\
Bt-2,12 & 13,4 & 0,19 & 3,24 & 8,46 & 58,6 & 2,38 & 1,29 \\
\hline
\end{tabular}


foi verificado quando sais monoquaternários de amônio com $1,3,4$ e 12 carbonos na cadeia foram intercalados em uma montmorilonita sódica ${ }^{[21]}$.

\section{Termogravimetria}

A Figura 4 apresenta as curvas de degradação térmica da bentonita sódica e dos complexos Bt-ioneno. Os complexos apresentam curvas de degradação semelhantes entre si, provavelmente, por se tratarem de polímeros de uma mesma família diferenciados apenas pelo número de grupos metilênicos na cadeia carbônica. No primeiro estágio de decomposição, entre 25 e $100{ }^{\circ} \mathrm{C}$, ocorre uma perda de massa maior para a bentonita sódica $(\approx 10 \%)$, correspondente à remoção de água das intercamadas coordenadas ao $\mathrm{Na}^{+}$. Este fato é um indicativo de que os complexos absorvem menos água, devido a suas propriedades organofílicas, como foi observado em argilas tratadas com sais monoquaternários de amônio numa seqüência crescente de número de carbono na cadeia do sal[ ${ }^{[21]}$. A perda de massa na faixa de 100 a $600^{\circ} \mathrm{C}$ para Bt-Na foi de aproximadamente $5 \%$, relativa à degradação da matéria orgânica. Esse valor está coerente com os resultados de fluorescência de raios X que indicaram um percentual de $6 \%$ de carbono. Nessa mesma faixa de temperatura $\left(100\right.$ a $\left.600{ }^{\circ} \mathrm{C}\right)$ a perda de massa para os complexos Bt-ionenos é bem maior, variando de $15 \%$ para o complexo Bt-EPIDMA até $20 \%$ para Bt-2,10 ioneno. Essas perdas de massa são originadas da oxidação e decomposição dos policátions intercalados e de água adsorvida abaixo de $220^{\circ} \mathrm{C}$, cuja quantidade diminui com o aumento do espaçador do ioneno, possivelmente devido ao aumento da hidrofobicidade. Através das curvas de DTG (não mostradas), foi possível verificar que esses materiais apresentam uma estabilidade térmica relativamente alta. Em todos os casos, o início da degradação térmica só é observado acima de $200^{\circ} \mathrm{C}$. Os complexos Bt-ionenos são mais estáveis termicamente que o complexo Bt-EPI-DMA em função do tipo de contra-íon, estrutura e conformação. Dentre os complexos Bt-ionenos, a estabilidade térmica cresce com o aumento do tamanho do espaçador do ioneno, cuja degradação pode gerar unidades monoméricas ou estruturas alifáticas de baixa massa molar ${ }^{[22]}$.

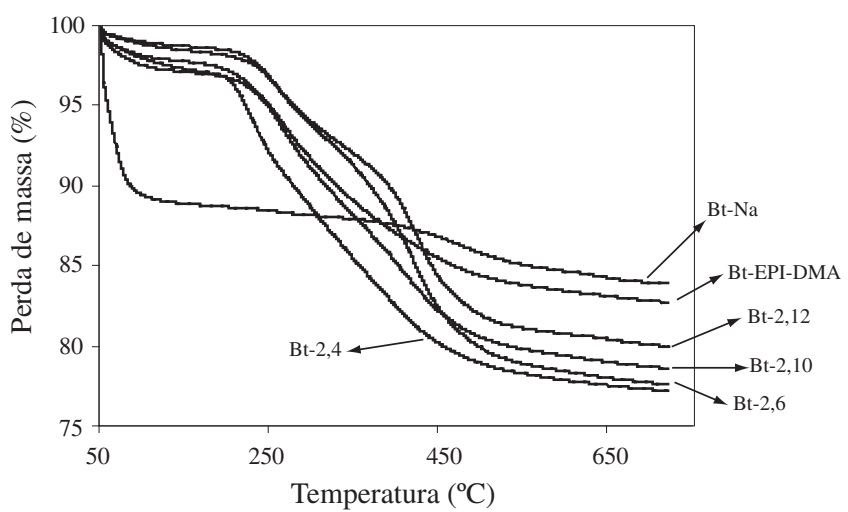

Figura 4. Curvas de termogravimetria para Bt-Na e os complexos Btionenos

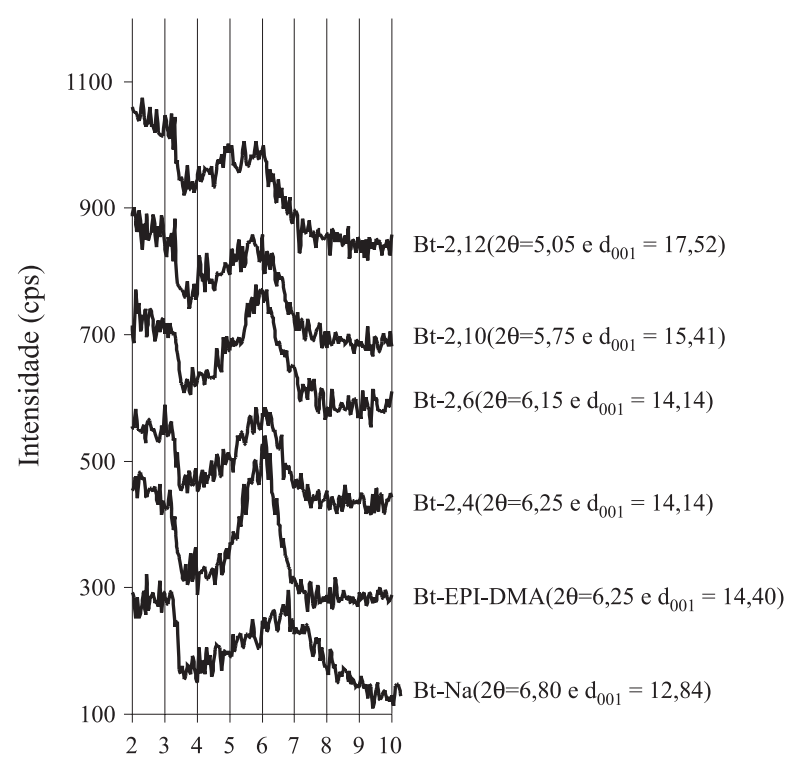

Figura 5. Difratogramas de raios X para Bt-Na e os complexos Bt-ionenos

\section{Espectroscopia fotoeletrônica de raios $\mathbf{X}$}

Para verificar a intercalação dos policátions nas intercamadas da argila, a Bt-Na e os complexos Bt-ionenos foram analisados por difração de raios $\mathrm{X}$. Os valores de espaçamentos basais (Figura 5) foram obtidos pela equação de Bragg[ ${ }^{[23]}$.

Os valores obtidos de espaçamentos basais, observados à temperatura ambiente, refletem o modo de adsorção do polímero. Os picos deslocaram-se para valores menores de ângulo $2 \theta\left(^{\circ}\right)$ com o aumento do tamanho do espaçador do ioneno, indicando um aumento na distância das intercamadas da argila. Esse resultado mostra que a adsorção desses ionenos não teve qualquer influência da massa molar, mas sim da densidade de cargas na cadeia do polímero, isto é, quanto menor a densidade de carga, mais "laços" são formados e maior é a expansão basal observada entre as camadas da argila.

$\mathrm{O}$ valor de espaçamento basal obtido para o Bt-EPI-DMA foi de 14,14 $\AA$, o que está de acordo com resultados já reportados na literatura ${ }^{[15]}$. Como esperado, os valores de espaçamento basais obtidos para os complexos Bt-2,4 e Bt2,6 ionenos, com menores tamanhos de espaçador, ficaram bem próximos do valor obtido para o Bt-EPI-DMA. Esses valores mais baixos de espaçamento basal refletem o maior número de segmentos trens do policátion na superfície da argila.

Embora todos esses policátions sejam 100\% catiônicos, foram observados os maiores valores de espaçamento basal para os 2,10- e 2,12-ioneno $\left(\mathrm{d}_{001}=15,41 \AA\right.$ e $\mathrm{d}_{001}=17,52 \AA$, respectivamente).A maior distância entre os centros de carga se reflete em maiores números de "laços" e "caudas" não adsorvidos ao longo da superfície da argila, fazendo com que suas camadas, originalmente com $\mathrm{d}_{001}=13,00 \AA$, entrem em expansão.

Quando comparados entre si, os ionenos têm suas densidades de carga diminuída com o aumento no tamanho da 
cadeia do espaçador. As quantidades livres de superfícies de siloxano (que é hidrofóbica), ocorrendo entre os sítios quaternários de amônio vizinhos, são maiores nas argilas tratadas com os polímeros tendo as menores densidades de carga. Essa quantidade de superfície livre de siloxano é importante para a interação e acomodação de algumas moléculas de poluentes orgânicos ${ }^{[15]}$.

Esses dados indicam que é possível produzir bentonitas modificadas com ionenos, cujos espaçamentos basais podem ser alterados em função da adsorção desses policátions lineares, variando-se o tamanho de seus espaçadores parafínicos $\mathrm{e}$, conseqüentemente, suas densidades de carga.

\section{Conclusões}

Os ionenos foram sintetizados e caracterizados quanto a suas massas molares por análise de ressonância magnética nuclear de hidrogênio

A adsorção dos ionenos na bentonita foi evidenciada principalmente pela troca catiônica $\left(\mathrm{Na}^{+} \mathrm{e} \mathrm{Ca}{ }^{+2}\right)$ em altos valores, acima da CTC da argila, além das perdas de massa verificadas por análise térmica e o surgimento de bandas de absorção na região do infravermelho relativas à ligação $\mathrm{C}-\mathrm{H}$. Os espaços interlamelares foram alterados em função do tamanho do espaçador e não foram influenciados pelas massas molares. Embora todos os ionenos sejam $100 \%$ catiônicos, foi a distância entre os seus centros de carga que determinou os espaçamentos basais.

A modificação de bentonitas com esses policátions leva a um controle mais efetivo sobre o teor de carbono orgânico incorporado, a quantidade de segmentos em "laços" e "caudas" e a quantidade de superfície de siloxano disponível, indicando que esses materiais poderão ser utilizados como removedores de poluentes orgânicos. Além disso, esses policátions são facilmente sintetizados e não necessitam de modificações em sua estrutura para controlar o grau de hidrofobicidade, uma vez que esse parâmetro pode ser projetado na síntese do ioneno desejado.

\section{Agradecimentos}

À CAPES pelo suporte financeiro e à COPPE pelas análises de fluorescência e difração de raios X.

\section{Referências Bibliográficas}

1. Smith, J \& Galan, A. - Environmental Science and Technology, 29, p.685 (1995).

2. Dentel, S.K.; Bottero, J.Y.; Khatib, K.; Demougeot, H.; Duguet, J.P. \& Anselme,C. -Water Research, 29, p.1273 (1995)

3. Althen, G.R. - Waste Management., 8, p. 623 (1995).
4. Rodriguez-Sarmiento, D.C \& Pinzón-Bello, J.A., - Applied Clay Science, 18, p.173 (2001).

5. Smith, J.A.; Bartell-Hunt, S.L. \& Burns, S.E. - Journal of Hazardous Materials, B96, p.91 (2003)

6. Redding, A.Z.; Burns, S.E.; Upson, R.T. \& Anderson, E.F. - Journal of Colloid and Interface Science, 250, p.261 (2002).

7. Klumpp, E.; Heitmann, H. \& Schwuger, M.I. - Colloids Surface., A 78(1-3), p.93 (1993)

8. Lee, S.Y. \& Kim, S.J. - Applied Clay Science, 22, p.55 (2002).

9. Koh, S. \& Dixon.; J.B. - Applied Clay Science, 18, p.111 (2001)

10. Yildiz, N.; Kapucu, H. \& Çalimli, A. - Turkish Journal of Eng. Environmental. Sci., 27, p.397 (2003)

11. Breen, C. - Applied Clay Science., 15, p.187 (1999).

12. Durand-Piana, G.; LaFuma, F. \& Audebert, R. - Journal of Colloid and Interface Science., 119, p.47. (1987)

13. Breen, C.; Rawson, J.O. \& Mann, B.F. - Journal of Materials Chemistry., 6, p.253 (1996)

14. Churchman, G.J.- Applied Clay Science., 21, p.177( 2002).

15. Breen, C. \& Watson, R.- Journal of Colloid and Interface Science., 208, p.422 (1998)

16. Rembaum, A.; Baumgartner, W. \&, Einsenberg. A. Polymer Letters 6, p.159 (1968)

17. Rembaum, A. \& Noguchi, H. - Macromolecules 3, p.261 (1972).

18. Phillips, K.G.; Forest, R. \& Zarneck, W. E. - US 76/ 3,975,347, D (1976)

19. Noguchi, H. - Makromolecules Rapid Communication 13, p.185 (1992).

20. Farmer, V.C. - "The Infrared Spectra of Minerals". Farmer, London, (1974).

21. Bala, P.; Samantaray, B.K. \& Srivastava, S.K. - Materials Research Bulletin., 35, p.1717 (2000)

22. Berwig,E.; Severgnini, V.L.S.; Soldi, M.S.; Bianco,G.; Pinheiro,E.A.; Pires, A.T.N. \& Soldi,V.Polymer Degradation and Stability., 79, p.93 (2003)

23. Santos, P.S. - "Ciência e tecnologia de argilas", Edgard Blücher Ltda, São Paulo (1989).

Enviado: 05/10/04

Reenviado: $26 / 02 / 05$

Aprovado: 10/03/05 